\title{
Impact of Automated Vehicles Using Eco-Cruise Control on the Traffic Flow
}

\author{
Balázs Németh" ${ }^{1 *}$ Péter Gáspár¹, Zsuzsanna Bede ${ }^{2}$ \\ ${ }^{1}$ Institute for Computer Science and Control (SZTAKI), Eötvös Loránd Research Network (ELKH), H-1111 Budapest, \\ 13-17 Kende utca, Hungary \\ 2 Department of Control for Transportation and Vehicle Systems, Budapest University of Technology and Economics, \\ H-1111 Budapest, 2 Stoczek utca, Hungary \\ * Corresponding author, e-mail: balazs.nemeth@sztaki.hu
}

Received: 22 April 2021, Accepted: 28 April 2021, Published online: 15 December 2021

\begin{abstract}
The paper provides a detailed analysis of the impact of automated vehicles using eco-cruise control system on the traffic flow. The speed profiles of vehicles using eco-cruise control system generally differ from those of conventional human-driven vehicles. The characteristics of the traffic flow on macroscopic traffic level combine both automated and human-driven vehicles. In the simulation-based analysis the effects of traffic volume and the ratio of the automated vehicles are in the focus. Based on the results the analysis an extension of the eco-cruise control is also proposed, in which the balance between the traffic flow and transport efficiency is achieved.
\end{abstract}

\section{Keywords}

eco-cruise control, speed profile, macroscopic simulation, traffic flow

\section{Introduction}

The research and development of various energy-efficient strategies of automated vehicles have been in the focus of the automotive industry and research institutes (Sciarretta et al., 2015). One of these methods is called the eco-cruise control of vehicles, which focuses on the velocity profile. The purpose of the velocity control is to guarantee effective, comfortable, safe and economical traffic by exploiting the features of both vehicle dynamics and environment. There are some important examples: characteristic of fuel consumption, delivered cargo, road inclinations, speed limits, traffic flow, traffic forecast (Hellström et al., 2009; Gáspár and Németh, 2019).

The control solutions result in speed profiles of the automated vehicles, which is usually significantly different from the speed selection of the human drivers. Their reasons are that the control systems of the automated vehicles have information about the road ahead, e.g., the usage of the road capacity or the upcoming downhill terrain characteristics. At the same time, unfortunately, this preliminary information is not available for the human drivers. Thus, in a conventional cruise control the steady speed set by the driver must be maintained as long as it is necessary (Li et al., 2011). The widespread use of the eco-cruise control influences the traffic flow significantly. The most important task of the eco-cruise control is to find a balance between the requirement of an individual vehicle and its impact on the traffic flow.

Several papers have been published in the topic of eco-cruise control, which focus on the performances of an individual vehicle. A multi-criteria optimization between journey time and fuel consumption was proposed by (Saerens et al., 2013). A multi-point boundary-value task was defined by (Passenberg et al., 2009). Since in the formulation several types of performances are considered such as input constraints and nonlinearities of vehicle dynamics, the numerical solution of the task is difficult. The implementation of a receding horizon control on a truck to provide a possible solution was presented in (Hellström et al., 2009). This paper shows that depending on the cruise control speed setting the achieved traveling time might slightly increases. The multi-criteria tasks of the hybrid vehicles are to take into consideration the state of charge of the battery, the energy consumption and the navigation map, see e.g., (Ambühl and Guzzella, 2009).

Another task is the analysis of the vehicle-traffic interactions, in which vehicles using eco-cruise control system 
and human-driven vehicles are traveling together on the roads, see e.g., (Li and Ioannou, 2004), (Wang et al., 2019). The challenges of the mixed traffic analysis and control are in the focus of several research works. A stability analysis based on car-following models was presented in (Zhu and Zhang, 2018). This paper presents that the varying sensitivity and smooth factors of automated vehicles affect traffic flux. The mixed traffic flow, in which both car-following control and bilateral control are used simultaneously, was proposed by (Wang and Horn, 2020). A control strategy to achieve a smooth traffic flow was developed by (Zheng et al., 2020). A cooperative cruise control of multiple cars in mixed traffic, in which conventional vehicles are considered hybrid dynamical systems was examined by (Chin et al., 2015). The paper shows that the cooperative control improves overall stability. The impact of vehicles using eco-cruise control systems on the traffic flow was presented by (Lu et al., 2019). The impact of vehicles using eco-cruise control and speed control on traffic flow was proposed by (Gáspár and Németh, 2019).

The goal of this paper is to analyse the impact of automated vehicles using eco-cruise control system on the traffic flow. The speed profiles of the automated vehicles may differ from those of the conventional vehicles, thus the conventional characteristics of the traffic flow based on the fundamental diagram changes. The motivation of the paper is introduced from a microscopic point-of-view through a high-fidelity CarSim environment. A simulation-based analysis of the traffic flow is performed, in which the current traffic inflow on the road and the ratio of automated vehicles are considered. In the examination the microscopic traffic simulator VISSIM is applied. Based on the analysis an extension of the eco-cruise control method, which considers the results of the analysis, is proposed. Here the purpose is to create a balance between the traffic flow and energy-efficient traveling.

The paper is structured as follows. The basics of the eco-cruise control design and the effect of the automated vehicle on the surrounding vehicles are introduced in Section 2. It is the basis of the further examinations of the traffic flow. The impact of the automated vehicles on the traffic flow is detailed in Section 3. The operation of the eco-cruise control strategy together with the macroscopic system is presented in Section 4. Finally, the results of the paper are concluded.

\section{Impact of automated vehicles on the surrounding vehicles}

The purpose of this section is to illustrate the impact of the automated vehicles using eco-cruise control on the surrounding vehicles. In this microscopic point of-view the local impact of an individual vehicle is examined.

The eco-cruise control strategy which has been built in automated vehicles was presented in (Gáspár and Németh, 2019). The basis of the eco-cruise control is to consider road inclinations, speed limits and traffic information from a finite horizon ahead of the vehicle. The purpose of the method is to find a speed profile which provides a balance between the performances such as energy consumption and traveling time, while safe distances from the other vehicles are guaranteed. It leads to a multi-criteria optimization task.

The result of the optimization is a varying scaling factor $R_{1} \in\{0,1\}$. The factor $R_{1}$ provides a priority between energy consumption and traveling time. Its large value is related to the importance of minimizing energy consumption, while its small value is related to the importance of minimizing total travel time. In practice $R_{1, \max }<1$ is selected in order to avoid significant variations in the required speed. Moreover, in the local traffic the speed of the vehicle must be selected to guarantee the safe distances from the surrounding vehicles. The optimization task is Eq. (1):

$\max _{\left[0, R_{1, \max }\right]} R_{1}$,

where $R_{1, \max }$ is a constant parameter, while $R_{1}$ varies dynamically.

In the optimization task (1) several constraints must be considered. The selection is influenced by the speeds of the surrounding vehicles and their locations. The main factors are the preceding and the following vehicles in the current lane $\left(R_{1, p}, R_{1, f}\right)$ and those in the adjacent lanes $\left(R_{1, p a}, R_{1, f a}\right)$. The possibility of the lane change of the automated vehicle in various situations is also part of the factors. For example, if the automated vehicle catches up with a preceding vehicle and there is no possibility of overtaking, the speed must adapt to the speed of the preceding vehicle. In this case a new $R_{1, p}$ is calculated and the speed of the preceding vehicle is set to a reference $v_{\text {lead }}$. Similarly, when the follower vehicle catches up with the automated vehicle, the speed of the automated must be adapted to that of the follower vehicle through a new $R_{1, f}$. In this way the obstacles 
in the motion of follower vehicle is avoided. Nevertheless, in both examples the designed speed may differ from the requested speed. It means that occupancies of both the current and the adjacent lanes must be analyzed. Based on a similar way, the factors $R_{1, p a}$ and $R_{1, f a}$ are calculated also in the adjacent lane. If the difference between the factors $R_{1, p}$, $R_{1, p a}, R_{1, f}, R_{1, f a}$ is higher than a predefined limit, the lane change maneuver is proposed to keep the economic aspect.

The resulting factor is applied in the computation of the optimal speed profile. The current reference speed $\lambda$ is defined by function $\mathbf{f}$ in the following way:

$$
\lambda=\mathbf{f}\left(Q\left(R_{1}, \alpha_{i}, v_{r e f, i}\right), \gamma_{i}\left(R_{1}, \alpha_{i}, v_{r e f, i}\right)\right),
$$

where $\alpha_{i}$ is the road slope and $v_{r e f, i}$ is the speed limit in segment $i \in\{1, . ., n\}$ ahead of the vehicle, where $n$ is the number of segments in the prediction horizon. The parameters $Q$ and $\gamma_{i}, i \in\{1, . ., n\}$ are formulated as convex combinations of $Q=1-R_{1}(1-\bar{Q})$ and $\gamma_{i}=R_{1} \bar{\gamma}_{i}+R_{2} \breve{\gamma}_{i}=R_{1} \bar{\gamma}_{i}$. The parameters $\bar{Q}$ and $\bar{\gamma}_{i}$ are the solutions of the quadratic optimization task.

\subsection{Simulation example of the interaction between the vehicles}

In the simulation example the impact of the eco-cruise control on the surrounding vehicles is illustrated through a CarSim simulation scenario. The automated vehicle travels in the outer lane of a highway, where a slower vehicle must be overtaken. The purpose of the scenario is to show that the maneuver can be performed without significantly disturbing the vehicles in the adjacent lane. There are two conflicts in the simulation. First, the overtaking maneuver cannot be performed, because there might be other vehicles in the inner lane. Since the vehicle in the adjacent lane has high speed, the automated vehicle must wait until the lane becomes free. Second, the automated vehicle changes the lane when it is free and other faster vehicle in the inner lane catches up with the automated vehicle. Thus, the automated vehicle must increase its own speed. When the vehicle in the adjacent lane at a sufficient distance behind, the automated vehicle completes the overtaking without significantly disturbing the vehicles in the inner lane.

The signals of the simulation are shown in Figs. 1 and 2. The speed of the automated vehicle is illustrated in Fig. 1(a). At the beginning of the scenario the vehicle is in the outer lane, and its speed is increased, see the actuation of the throttle in Fig. 1(b). The shifting procedure is controlled by a logic based on the speed and the load of the engine, which has impact on the variation of the throttle, see e.g.

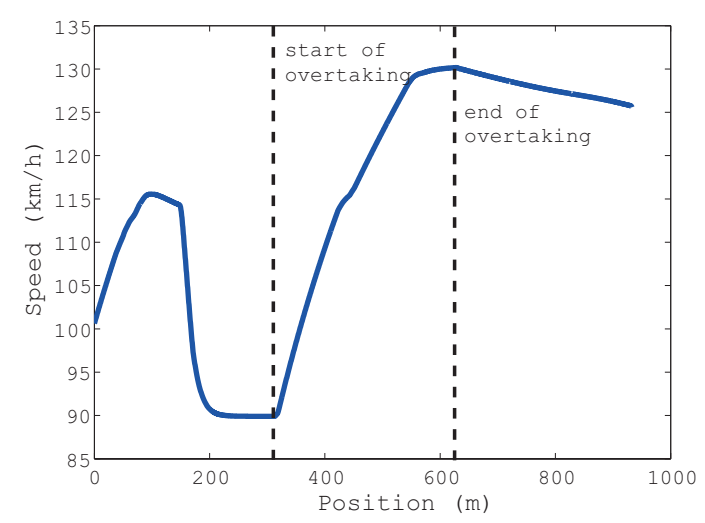

(a)

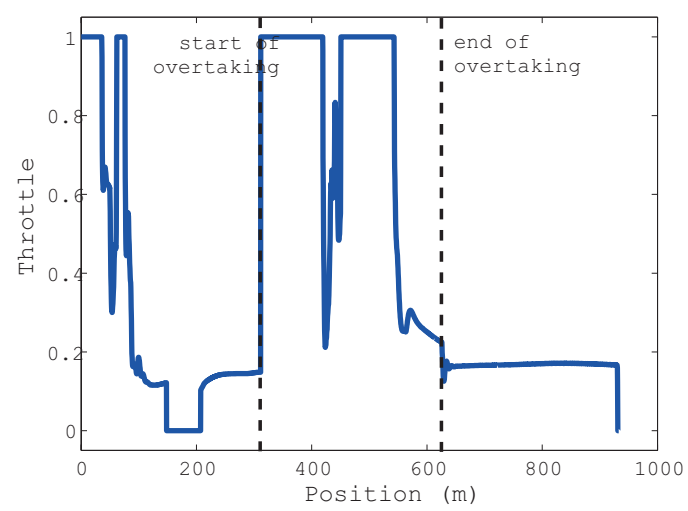

(b)

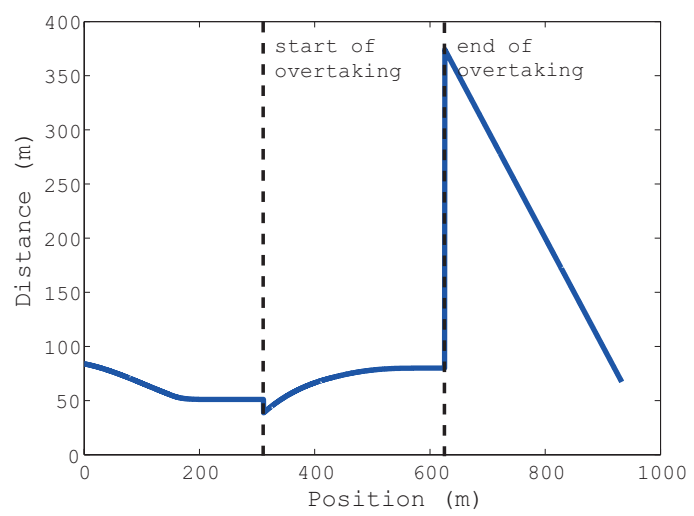

(c)

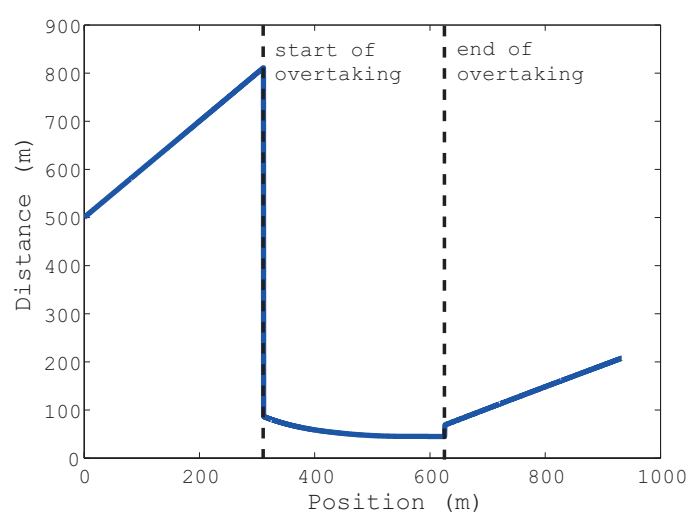

(d)

Fig. 1 Signals in the lane-change maneuver; (a) Speed; (b) Throttle; (c) Distance ahead of the vehicle; (d) Distance behind the vehicle 


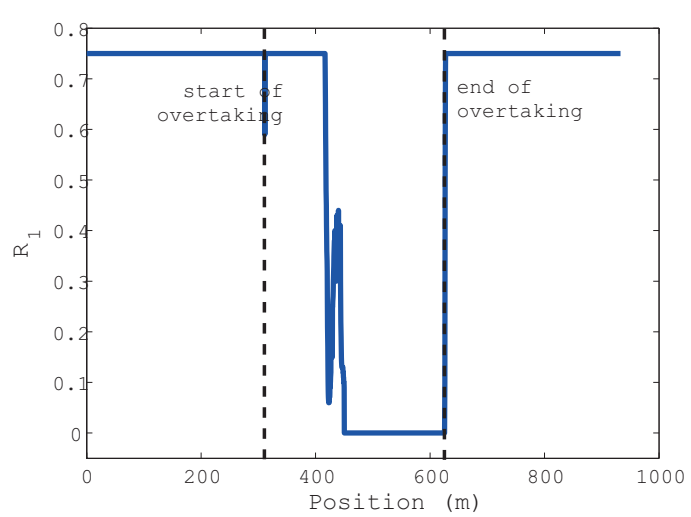

(a)

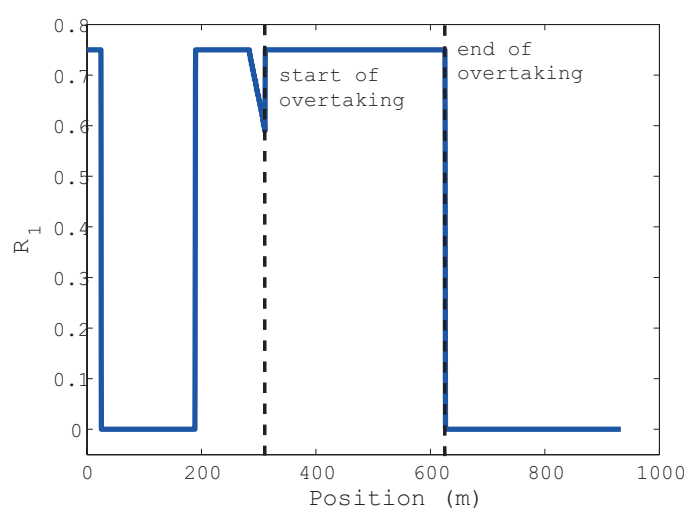

(c)

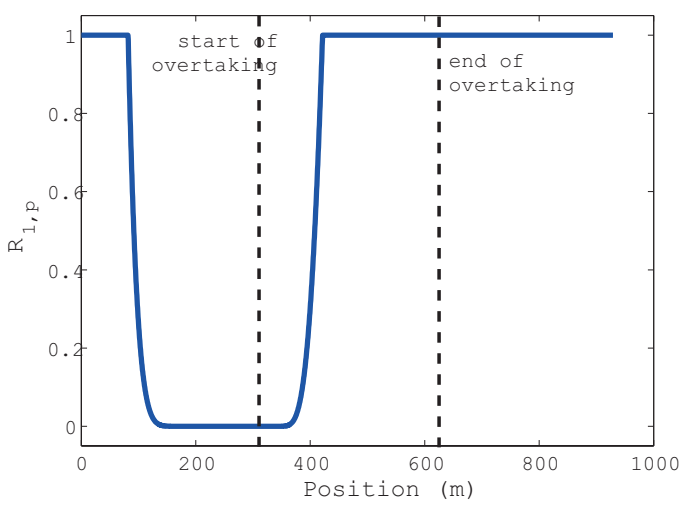

(b)

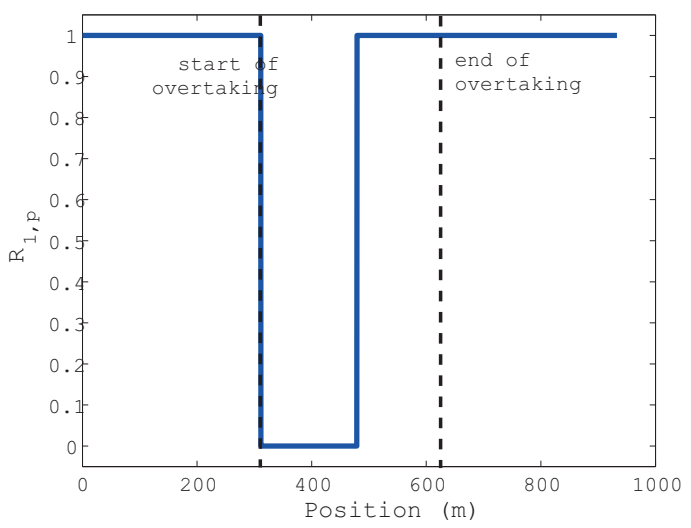

(d)

Fig. 2 Weight $R_{1}$ in the current and the adjacent lanes; (a) $R_{1, f}$ in the current lane; (b) $R_{1, p}$ in the current lane; (c) $R_{1, f a}$ in the adjacent lane; (d) $R_{1, p a}$ in the adjacent lane

between $0 \ldots 100 \mathrm{~m}$. Since the automated vehicle accelerates, it catches up with the preceding vehicle as the distance between the vehicles is shown (Fig. 1(c)). Due to the vehicle in the adjacent lane the overtaking maneuver cannot be performed, thus the signal $R_{1, p}$ is reduced (Fig. 2(b)). It results in the speed reduction to $v_{\text {lead }}=90 \mathrm{~km} / \mathrm{h}$, and the safe distance $d_{\text {safe }}=50 \mathrm{~m}$ is kept. Simultaneously, $R_{1, f a}=0$ is set, because there is another vehicle in the adjacent lane (Fig. 2(c)). When the vehicle in the adjacent lane overtakes the controlled vehicle, $R_{1, f a}=R_{1, \max }$, in which $R_{1, \max }$ is set, see at point $200 \mathrm{~m}$. Although in the simulation constant $d_{\text {safe }}$ is considered, the proposed cruise method is independent from a specified distance value, i.e. speed-dependent headway can also be incorporated in the method.

Since the speed of this vehicle is greater than that of the automated vehicle, $R_{1, p a}=0$ is set in the adjacent lane, see Fig. 2(d). Since the safe distance in the new lane cannot be guaranteed, the overtaking maneuver is not started. The overtaking and the lane change begin only at point $320 \mathrm{~m}$. When the overtaking maneuver has been carried out, the current lane will be the new one and the adjacent lane will be the original one. Thus, $R_{1, p}$ in the current lane is reduced to zero and $R_{1, p a}$ in the original lane is increased to 1 , because the distance between the automated vehicle and the overtaken vehicle is decreased, see Fig. 2(b) and 2(d). Furthermore, the speed is increased in order to achieve the designed speed, see Fig. 1(a) and the throttle signal Fig. 1(b).

In the second part of the simulation the follower vehicle catches up with the automated vehicle as shown in Fig. 1(d). When the distance between the automated vehicle and the follower vehicle decreases to the safe distance, weight $R_{1, f}$ is reduced in order to accelerate the automated vehicle, see Fig. 2(a) at point $410 \mathrm{~m}$. Thus, the speed of the automated vehicle is increased up to $130 \mathrm{~km} / \mathrm{h}$, as shown in Fig. 1(a). During the acceleration the vehicle of the adjacent lane is overtaken, which is shown by the signal $R_{1, p a}$ at point $480 \mathrm{~m}$ in Fig. 2(d). Since the automated vehicle is faster than the overtaken vehicle, $R_{1, f a}$ in the adjacent lane is set to $R_{1, \max }$, see Fig. 2(c). Based on this signal the lane change strategy suggests that the automated vehicle should return to the original lane. When the safe distance from the overtaken vehicle is reached, the overtaking maneuver has 
been finished and the controlled vehicle is driven back to the lane, see point $620 \mathrm{~m}$. Thus, the distance behind the vehicle increases, while the speed of the vehicle and the throttle are reduced significantly, see Fig. 1(a) and 1(b). Simultaneously, $R_{1, f}=R_{1, \max }$ in the new lane and $R_{1, f a}=0$ in the adjacent lane are set.

\section{Simulation-based examination from a macroscopic point-of-view}

The automated vehicle with the eco-cruise control is able to adapt to the surrounding vehicles, while their motion is not disturbed significantly. However, if there are a significant number of automated vehicles on the highway, they may have increased impact on the entire traffic flow. In this section a simulation-based analysis of the eco-cruise control vehicles on the mixed traffic with automated and human-driven vehicles is performed.

In the research the traffic model and the measurements of a test network are built in the VISSIM traffic simulator.

In the architecture of the simulation environment guarantees the traffic model and the graphical interface. It is connected to the Matlab software through the Component Object Model, in which the eco-cruise control is implemented. In the simulations two different ways of the reference vehicle speed selection are used. In the case of conventional vehicles, the reference speed of the vehicles is equal to the speed limit of the highway. All of the conventional vehicles received this information and set their own speed through a tracking control, see (Wiedemann, 1974). In the case of the eco-cruise controlled vehicles the reference speed trajectory is computed in Matlab through the optimization task. The result of the optimization is the current reference speed, which is provided to VISSIM and applied in the tracking control. In VISSIM the highway section is divided into sections. The number of the lanes, the grade and the orientation are set in every section. The topographic data and the trajectory of the highway are based on real values, which have been obtained from Google Earth.

In the demonstration example, a $20 \mathrm{~km}$-long 3-lane segment of the Hungarian M1 highway between Budapest and Tatabánya is modelled in VISSIM, in which the terrain characteristics (see Fig. 3) and the speed limits are taken into consideration. The speed limit on the section is $130 \mathrm{~km} / \mathrm{h}$, although there is a $90 \mathrm{~km} / \mathrm{h}$ limitation on the highway between $6-8 \mathrm{~km}$ segments. Using this model several simulations with different traffic inflows (500 veh/h $\leq$ qin $\leq 5000 \mathrm{veh} / \mathrm{h}$ with the sampling $500 \mathrm{veh} / \mathrm{h}$ ),

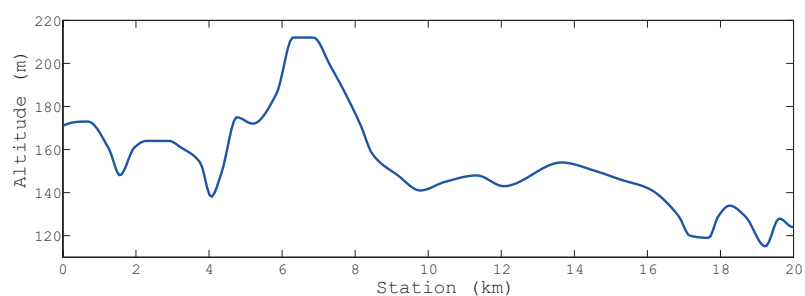

(a)

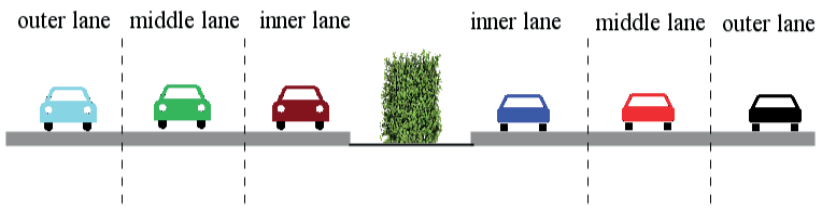

(b)

Fig. 3 Illustration of the M1 highway section; (a) Terrain characteristics of the highway section; (b) Sketch of the road

$\kappa$ values $(\kappa \in[1 \% ; 10 \% ; 20 \% ; 30 \% ; 40 \% ; 50 \%])$ are performed. The motivation behind the selection of these variables and parameter is based on the characteristics of the fundamental diagram. In Németh et al. (2017) it is proposed that the fundamental diagram depends on $\kappa$. Through the law of conservation and the fundamental relationship the dynamics of the traffic significantly depends on the characteristics of the fundamental diagram and the inflow of the highway section. In the following some examples from a detailed analysis illustrate the impact of the automated vehicles on the traffic flow.

\subsection{Simulation examples for the impact of the automated vehicles on the traffic flow}

In the first scenario the selected values are $q_{\text {in }}=3000 \mathrm{veh} / \mathrm{h}$ and $\kappa=1 \%$. Since there are low number of automated vehicles with eco-cruise control on the highway, the results of this scenario are close to the contribution of microscopic approach: the impact of the automated vehicles on the entire traffic flow is negligible, the traffic flow volumes in all lanes are smooth, see Fig. 4(a).

The traction forces of the conventional and the automated vehicles are illustrated in Fig. 4(b), where the green points are related to the conventional vehicles, while the red points represent the traction force of the automated vehicles. The results show that the traction force values of the eco-cruise controlled vehicles differ from those of conventional vehicles. The automated vehicles are able to realize their optimal speed profile, which results in force reduction on the entire route.

In the second scenario the ratio of the eco-cruise controlled vehicles is increased to $\kappa=20 \%$. Consequently, 


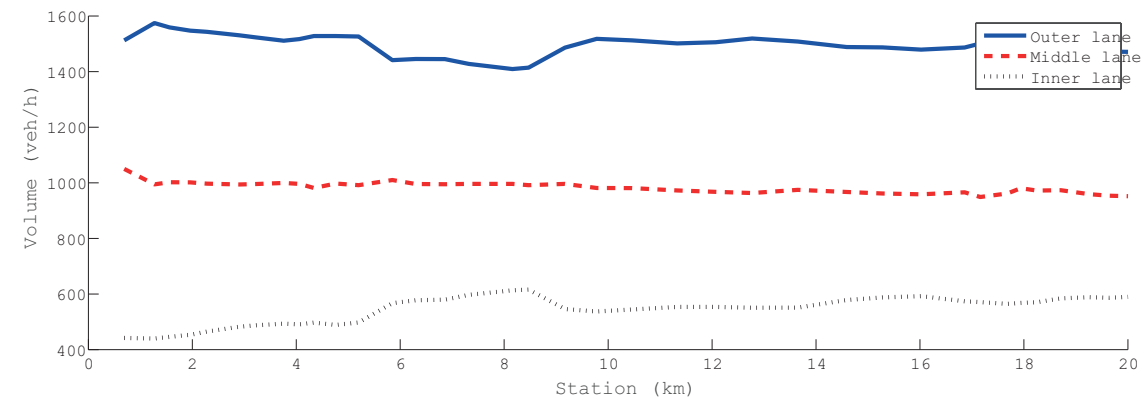

(a)

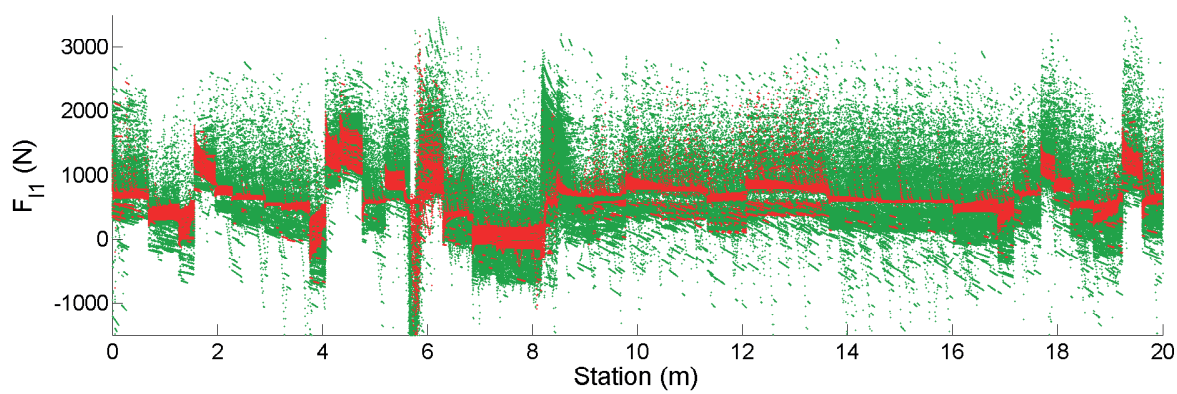

(b)

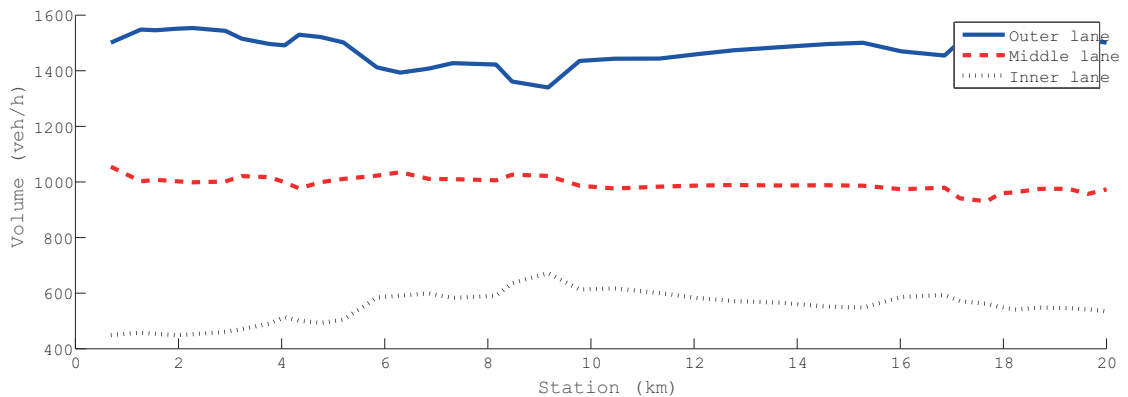

(c)

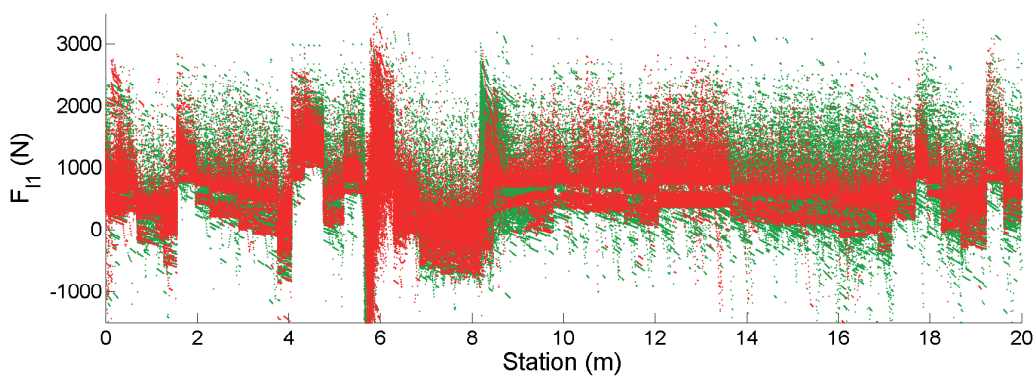

(d)

Fig. 4 Scenario 1: $q_{i n}=3000 \mathrm{veh} / \mathrm{h}, \kappa=1 \%$, Scenario $2: q_{i n}=3000 \mathrm{veh} / \mathrm{h}, \kappa=20 \%$; (a) Traffic flow volume (Scenario 1); (b) Traction force (Scenario 1); (c) Traffic flow volume (Scenario 2); (d) Traction force (Scenario 2)

there are a significant number of automated vehicles in the traffic flow, which results in a statistically increased number of interactions between the controlled and the other vehicles. The interactions (e.g. overtaking, deceleration maneuvers) result in the modification of their optimal speed profile. Due to the adaptation of the vehicles to each other the automated vehicles have impact on the traffic flow. Fig. 4(c) shows that the traffic volume in the lanes significantly varies. E.g., in the outer lane the volume is reduced, which means that the automated vehicles more often travel in the outer lane.

The effect of the higher $\kappa$ on the traction forces is illustrated in Fig. 4(d). It can be seen that the traction forces of the eco-cruise controlled vehicles are closer to those of 
the conventional vehicles. Thus, due to the increased traffic, not all of the eco-cruise controlled vehicles are able to guarantee the fuel-economy motion. It is in strong connection with the increased number of vehicle interactions. Furthermore, the energy consumption of the conventional vehicles slightly decrease. Thus, in this scenario the automated vehicles have a low impact on the traffic flow: the speed profile and the traction force of the vehicles without eco-cruise control are not modified significantly.

In the third simulation scenario $\kappa$ is increased to $50 \%$, which has a more increased impact on the traffic flow. The traffic flow volume varies to the Scenario 1 in all lanes, especially in the outer and in the inner lanes, see Fig. 5(a). The traction forces of all vehicles in the traffic flow decrease, as shown in Fig. 5(b). This scenario illustrates that the energy consumption of the traffic flow can be influenced by using a high $\kappa$ ratio. When the results are compared to the first scenario, which contains $99 \%$ conventional vehicles, the mean of the energy consumption of the conventional vehicles decreases from $13.562 \mathrm{~kJ}$ to $13.468 \mathrm{~kJ}$.

In the fourth scenario the impact of $q_{i n}$ on the traffic flow is illustrated. It means that the inflow increases to $q_{\text {in }}=5000 \mathrm{veh} / \mathrm{h}$ value, which represents rush hour traffic. Fig. 5(c)-(d) presents the results of this scenario. The consequence of the rush hour traffic is the adaptation of the eco-cruise controlled and the conventional vehicles to each other, which results in the characteristics of the traffic flow volume. Since the motion of the automated vehicles is closer to the conventional vehicles, it leads to a slight increase in their traction force, compared to Scenario 2. Moreover, the motion of the conventional vehicles also varies, which results in their force reduction by $2.3 \%$ also compared to Scenario 2. Thus, in heavier traffic the ecocruise control has a significant impact on all the vehicles in terms of force requirement.

The average energy consumption of the vehicles is examined based on the results of the macroscopic simulations, see Fig. 6. The results show that the eco-cruise controlled vehicles have lower energy consumption compared to the conventional vehicles. Moreover, the energy consumption of the conventional vehicles decreases with the increase of $\kappa$ and $q_{i n}$.

\section{Contributions of the macroscopic simulations in the eco-cruise control}

Automated vehicles have an impact on the entire traffic flow through their influences on the surrounding vehicles. Due to the increasing ratio of the automated vehicles in the traffic flow, this effect has a cumulative property. Consequently, the function $R_{1}$ must be designed to consider the requirements on the traffic flow. In this section the results of the macroscopic simulations are summarized and then, the contributions are built in the speed profile design of the automated vehicles.

The following conclusions can be defined by using the impact of $\kappa$ and $q_{\text {in }}$ on the traffic flow, see Section 3.

The increase in the traffic flow can lead to the reduction of the traction forces of the conventional and the eco-cruise controlled vehicles simultaneously. However, it increases the traveling time of the vehicles due to the dense traffic.

If the ratio $\kappa$ of the eco-cruise controlled vehicles increases, it is slightly disadvantageous for the motion of the eco-cruise controlled vehicles, but it improves the force reduction of the conventional vehicles.

The variation of $q_{\text {in }}$ and $\kappa$ has benefits on the entire traffic flow. It is necessary to find a balance between the contradictory effects, which is achieved by the selection of $R_{1}$, see (Gáspár and Németh, 2019). Since in the selection strategy of $R_{1}$ various safety and economy requirements have already been incorporated (see Section 2), the effects of $q_{\text {in }}$ and $\kappa$ are considered in the determination of $R_{1, \max }$. Its advantage is that the safety constraints of the eco-cruise control are not influenced by the modification of $R_{1, \max }$.

The appropriate selection of $R_{1, \max }$ the effect of the high $q_{\text {in }}$ on the traffic speed can be reduced, because the speed of the automated vehicle is closer to that of the conventional vehicle. As a result, the adaptation of the eco-cruise control vehicles to the conventional vehicles leads to an increase in the traffic speed. Furthermore, if $\kappa$ has a high value, the motion of the conventional vehicle can be significantly inhibited, which can be avoided with the limitation of $R_{1, \max }$. The assumptions of $q_{\text {in }}$ and $\kappa$ are formulated in a function, such as:

$R_{1, \max }=\mathbf{f}\left(q_{\text {in }}, \kappa\right)$,

where $\mathbf{f}$ is an appropriately chosen function.

f depends on the current road section and the traffic requirements. Fig. 7 shows a polynomial function example, which is generated through the numerical results of the simulation scenarios in Section 3. In the example function $R_{1, \max }$ is formulated as a polynomial $5^{\text {th }}$ order in and $2^{\text {nd }}$ order in $q_{i n}$. The selection method of the parameters of the polynomial is based on the results of the macroscopic simulations. There are several combination of $\kappa, q_{\text {in }}$ and $R_{1}$ pairs which are proposed to be reference values for $R_{1, \max }$. Then, the parameters of the polynomial are calculated 


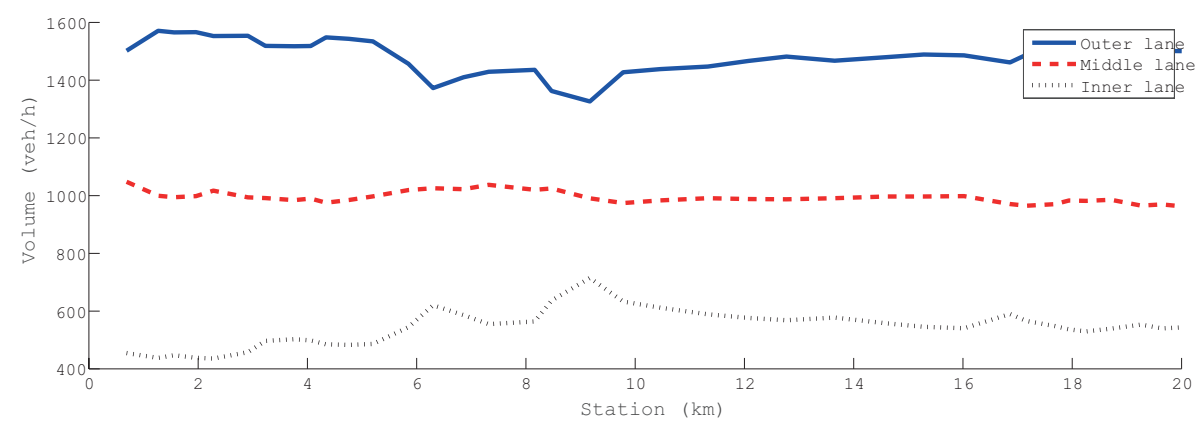

(a)

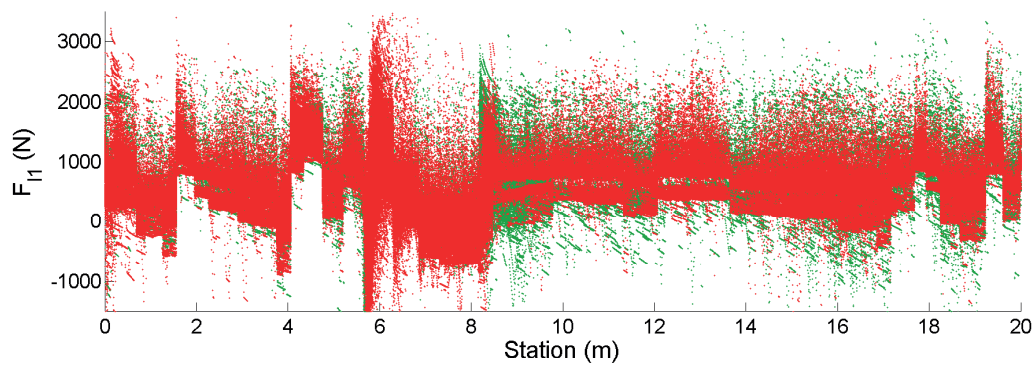

(b)

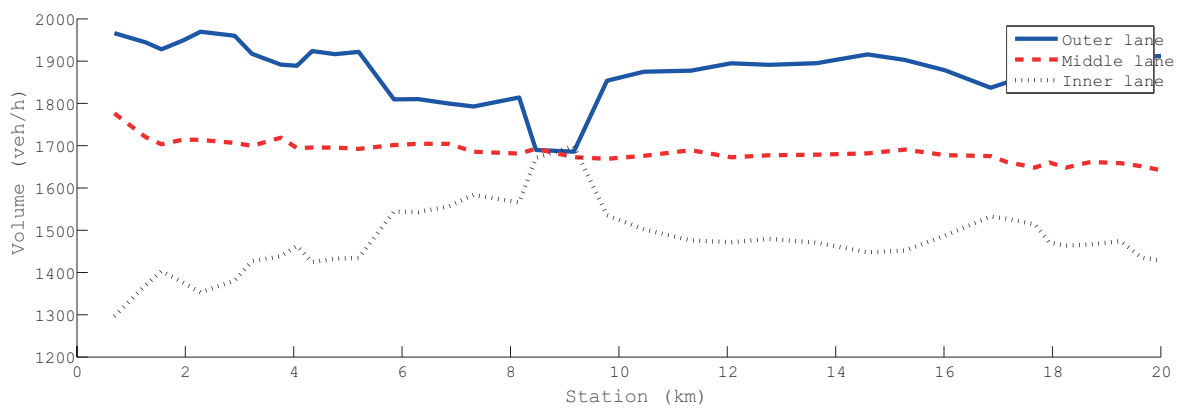

(c)

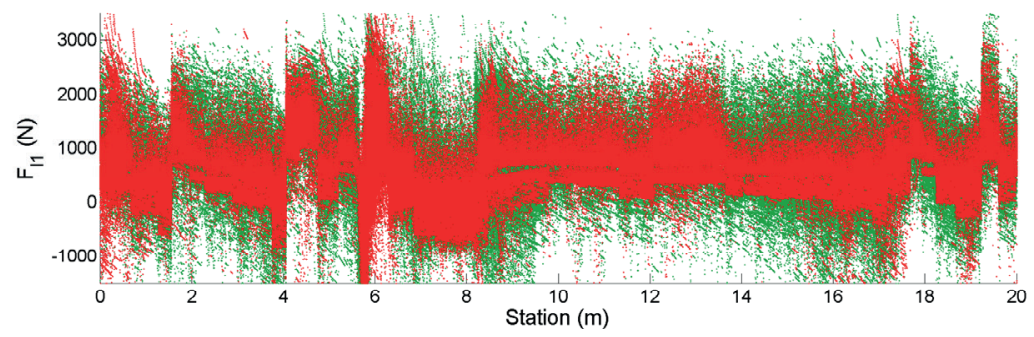

(d)

Fig. 5 Scenario 3: $q_{\text {in }}=3000 \mathrm{veh} / \mathrm{h}, \kappa=50 \%$, Scenario 4: qin=5000 veh/h, $\kappa=20 \%$; (a) Traffic flow volume (Scenario 3); (b) Traction force (Scenario 3); (c) Traffic flow volume (Scenario 4); (d) Traction force (Scenario 4)

based on least squares method, which guarantees that the resulting values of $R_{1, \max }$ fit on the reference values. The function indicates that at low $\kappa$ and $q_{\text {in }}$ values the high $R_{1, \max }$ value is preferred, which results in saving energy. If $\kappa$ or $q_{\text {in }}$ values increase, $\mathbf{f}$ is reduced. However, the inequality $R_{1, \max }>0, \forall \kappa, N$ must be guaranteed to improve the energy efficiency of the traffic flow.

\subsection{Simulation example on the automated vehicle}

In the final simulation example, the automated vehicle travels along a $30 \mathrm{~km}$ long section of the German highway $A 8$ between Ulm and Stuttgart. The terrain characteristics of the road are based on the data of Google Earth as it is illustrated in Fig. 8(a). The purpose of the simulation is to show that the variations of $q_{i n}$ and $\kappa$ are able to influence the speed profile of the vehicle through $R_{1, \max }$. 


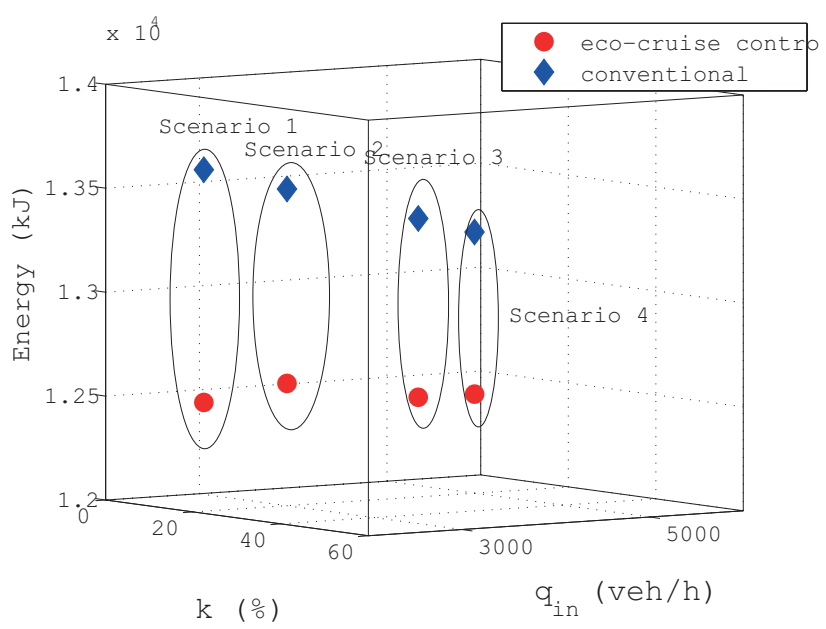

Fig. 6 Comparison of energy consumptions

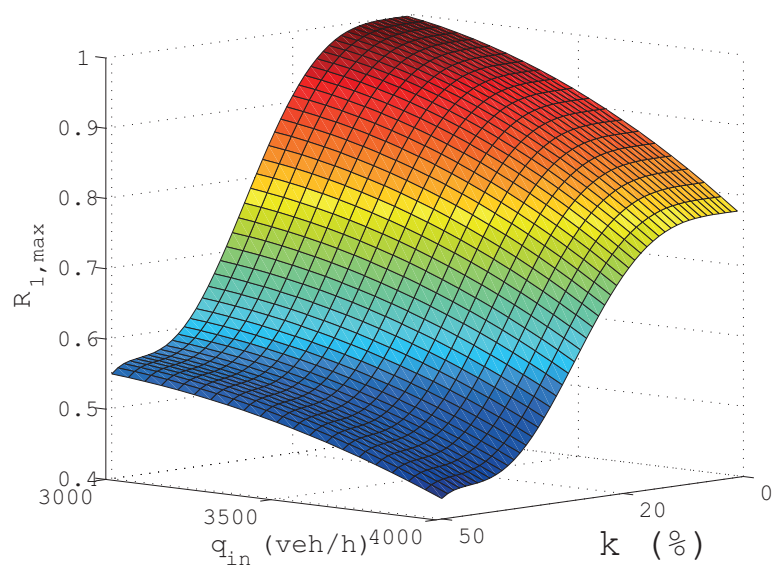

Fig. 7 Selection of the function $\mathbf{f}\left(q_{i n}, \kappa\right)$

The traffic flow at the entrance of the section is illustrated in Fig. 8(b). The simulation presents a scenario, which is the beginning of the rush-hour on the highway and the mean of the inflow volume increases continuously. The ratio of the automated vehicles on the highway section slowly varies in the simulation. The value of $\kappa$ is updated in every $50 \mathrm{~s}$ as it is shown in Fig. 8(c). The values of $q_{\text {in }}$ and $\kappa$ influence $R_{1, \max }$ based on the previous rules, see its variation in $8(\mathrm{~d})$. It has impact on the current speed of the automated vehicle (Fig. 8(e)). For example, between $300 \mathrm{~s} . . .370 \mathrm{~s}$ the value of $\kappa$ is decreased by approximately $10 \%$, which means that the eco-cruise control can prioritize energy saving. Thus, $R_{1, \max }$ is increased, which enforces the reduction of the fuel consumption. The speed profile is adapted to the terrain characteristics, which leads to the slight reduction in speed. Moreover, $q_{\text {in }}$ also has impact on the speed profile. In the time sections $100 \mathrm{~s} \ldots 150 \mathrm{~s}$ and $800 \mathrm{~s} \ldots 850 \mathrm{~s}$ the ratio $\kappa$ has the same value, while the mean of $q_{\text {in }}$ is increased with $1000 \mathrm{veh} / \mathrm{h}$ due to the

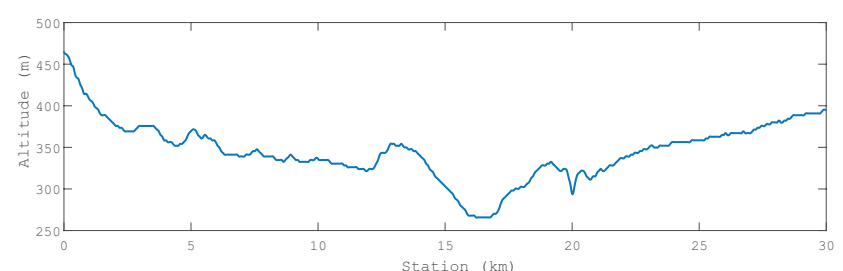

(a)

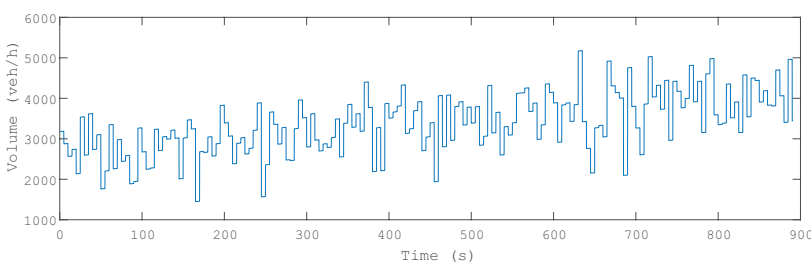

(b)

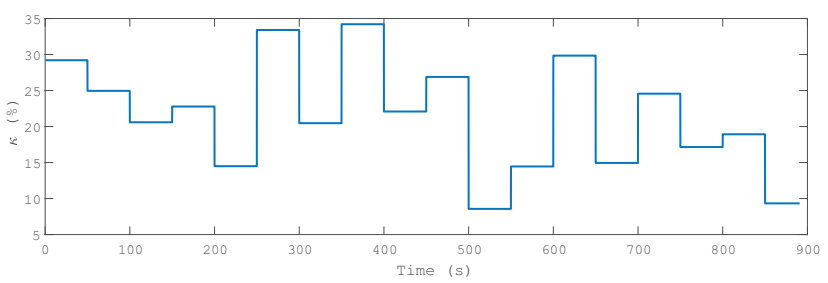

(c)

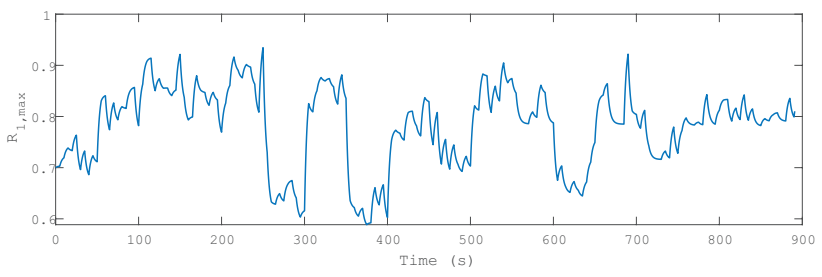

(d)

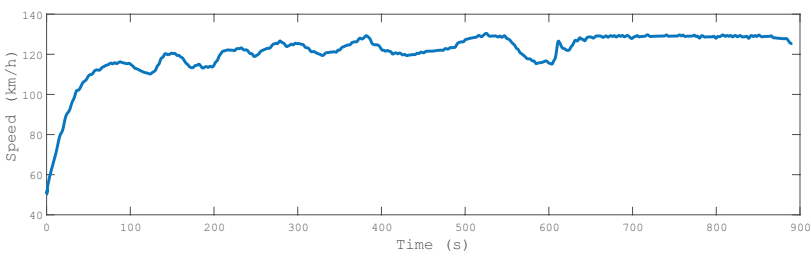

(e)

Fig. 8 Simulation with the improved eco-cruise control; (a) Altitude of the highway; (b) Traffic inflow volume; (c) Ratio of the automated vehicles; (d) $R_{1, \max }$; (e) Speed profile

rush-hour traffic. The avoidance of the significant traffic volume reduction requests increased speed profiles at higher $q_{\text {in }}$. Thus, $R_{1, \max }$ must be reduced, whose reduction has the value around 0.07 . It leads to $5 \mathrm{~km} / \mathrm{h} \ldots 10 \mathrm{~km} / \mathrm{h}$ increase in the speed profile, see Fig. 8(e).

\section{Conclusions}

In the paper the impact of automated vehicles using ecocruise control system on the traffic flow has been analyzed. The task is examined from both microscopic and 
macroscopic point-of-views. Different simulation scenarios are performed using the VISSIM traffic environment, in which the motions of the eco-cruise control vehicles and the conventional vehicles are traveling together. The volume of vehicles in the traffic network and the ratio of eco-cruise control vehicles in the traffic vary during the analysis. It is shown that these factors have significant impact on the traffic flow. Furthermore, an improved ecocruise control strategy is built into automated vehicles, in which their impact on the traffic flow is incorporated. The method guarantees an energy-efficient motion of the automated vehicles in the traffic, while an extreme variation of the traffic flow volume can be avoided.

\section{References}

Ambühl, D., Guzzella, L. (2009) "Predictive Reference Signal Generator for Hybrid Electric Eehicles", IEEE Transactions on Vehicular Technology, 58(9), pp. 4730-4740. https://doi.org/10.1109/TVT.2009.2027709

Chin, H., Okuda, H., Tazaki, Y., Suzuki, T. (2015) "Model predictive cooperative cruise control in mixed traffic", In: IECON 2015 - 41st Annual Conference of the IEEE Industrial Electronics Society, Yokohama, Japan, pp. 3199-3205. https://doi.org/10.1109/IECON.2015.7392593

Gáspár, P., Németh, B. (2019) "Predictive Cruise Control for Road Vehicles Using Road and Traffic Information", Springer,Cham,Switzerland. https://doi.org/10.1007/978-3-030-04116-8

Hellström, E., Ivarsson, M., Åslund, L., Nielsen, L. (2009) "Look-ahead control for heavy trucks to minimize trip time and fuel consumption", Control Engineering Practice, 17(2), pp. 245-254. https://doi.org/10.1016/j.conengprac.2008.07.005

Li, K., Ioannou, P. (2004) "Modeling of traffic flow of automated vehicles", IEEE Transactions on Intelligent Transportation Systems, 5(2), pp. 99-113. https://doi.org/10.1109/TITS.2004.828170

Li, S., Li, K., Rajamani, R., Wang, J. (2011) "Model Predictive MultiObjective Vehicular Adaptive Cruise Control", IEEE Transactions on Control Systems Technology, 19(3), pp. 556-566. https://doi.org/10.1109/TCST.2010.2049203

Lu, C., Dong, J., Hu, L., Liu, C. (2019) "An Ecological Adaptive Cruise Control for Mixed Traffic and Its Stabilization Effect", IEEE Access, 7, pp. 81246-81256. https://doi.org/10.1109/ACCESS.2019.2923741

Németh, B., Bede, Z., Gáspár, P. (2017) "Modelling and analysis of mixed traffic flow with lookahead controlled vehicles", IFAC PaperOnLine, 50(1), pp. 15639-15644. https://doi.org/10.1016/j.ifacol.2017.08.2384

Passenberg, B., Kock, P., Stursberg, O. (2009) "Combined time and fuel optimal driving of trucks based on a hybrid model", In: 2009 European Control Conference (ECC), Budapest, Hungary, pp. $4955-4960$.

https://doi.org/10.23919/ECC.2009.7075185

\section{Acknowledgement}

The research was supported by the Ministry of Innovation and Technology NRDI Office within the framework of the Autonomous Systems National Laboratory Program.

The research was supported by the Hungarian Government and cofinanced by the European Social Fund through the project "Talent management in autonomous vehicle control technologies" (EFOP-3.6.3-VEKOP-16-2017-00001).

The work of Balázs Németh was partially supported by the János Bolyai Research Scholarship of the Hungarian Academy of Sciences and the ÚNKP-20-5 New National Excellence Program of the Ministry for Innovation and Technology from the National Research, Development and Innovation Fund.

Saerens, B., Rakha, H. A., Diehl, M.,Van den Bulck., E. (2013) "A methodology for assessing eco-cruise control for passenger vehicles", Transportation Research Part D: Transport and Environment, 19, pp. $20-27$. https://doi.org/10.1016/j.trd.2012.12.001

Sciarretta, A., De Nunzio, G., Ojeda, L. L. (2015) "Optimal Ecodriving Control: Energy-Efficient Driving of Road Vehicles as an Optimal Control Problem", IEEE Control Systems Magazine, 35(5), pp. 71-90.

https://doi.org/10.1109/MCS.2015.2449688

Wang, J., Peeta, S., He, X. (2019) "Multiclass traffic assignment model for mixed traffic flow of human-driven vehicles and connected and autonomous vehicles", Transportation Research Part B: Methodological, 126, pp. 139-168. https://doi.org/10.1016/j.trb.2019.05.022

Wang. L., Horn, B. K. P. (2020) "On the Stability Analysis of Mixed Traffic with Vehicles Under Car-Following and Bilateral Control", IEEE Transactions on Automatic Control 65(7), pp. 3076-3083. https://doi.org/10.1109/TAC.2019.2945888

Wiedemann, R. (1974) "Simulation des Strassenverkehrsflusses" (Title in English), Institut für Verkehrswesen der Universität Karlsruhe, Karlsruhe, Germany. (in German)

Zheng, Y., Wang, J., Li, K. (2020) "Smoothing Traffic Flow via Control of Autonomous Vehicles", IEEE Internet of Things Journal,7(5), pp. 3882-3896.

https://doi.org/10.1109/JIOT.2020.2966506

Zhu, W-X., Zhang, M. H. (2018) "Analysis of mixed traffic flow with human-driving and autonomous cars based on car-following model", Physica A: Statistical Mechanics and its Applications, 496, pp. 274-285.

https://doi.org/10.1016/j.physa.2017.12.103 